\title{
Electromyography by Dr. Molina leon
}

\author{
Angel Molina León* \\ Specialist in Clinical Neurophysiology, Spain
}

Submission: October 27, 2017; Published: November 16, 2017

*Corresponding author: Ángel Molina León, Doctors and Nurses the Clinic Neurophysiology Service, Santa Lucia Hospital, Cartagena, Murcia, Spain

\section{Introduction}

(Figure 1) What is Electromyography and what is used?

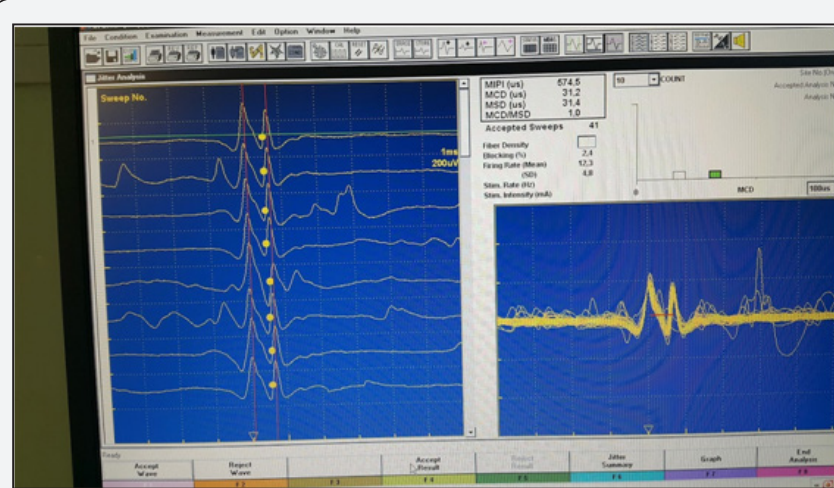

Figure 1

Electromyography (EMG) is the electrophysiological study of the neuromuscular system. It is a complementary test, and prolongation of the clinical neurological study. Given the broad neuromuscular system and brain, in Spain there is a specific and specific pathway via MIR of Neuromuscular which is called Clinical Neurophysiology which includes EEG, EMG, Multimodal Potentials (PEAT, PEV, PESS) and Intraoperative Monitoring. In addition to sleep pathology, and polysomnography and is taught by physicians via MIR with 4 years of training within this specialty. The EMG is designed in each case according to each protocol, according to the specific pathology, it is not the same to see a syndroem of the carpal tunnel that to see a Polyneuropathy or to study in depth a patient with suspicion of lateral sclerosis amiotrofica ELA, and in function of this each protocol has a different duration. One of the characteristics in my judgment of a good neurophysiologist is meticulousness, patience, the taste for this part of medicine and objectivity, it is very important to be objective.

\section{The test consists of two part electroneurography and electromyography}

\section{Indications of electromyography:}

a) Differentiation between weakness of central or peripheral origin. b) Differentiation between weakness of neurogenic or myogenic origin.

c) Differentiation between preganglionic (radicular) or postganglionic (plexular / truncal) lesion.

d) Location of the lesion in the mononeuropathies compressive or traumatic and determination of the degree of affectation (focal demyelination versus axonal degeneration).

e) Differentiation between multifocal neuropathies and polyneuropathies; degree of involvement of the motor and sensory fibers.

f) Differentiation between demyelinating and axonal neuropathies.

g) Determination of prognosis in neuropathies.

h) Characterization of neuromuscular junction disorders (pre- or postsynaptic).

i) Identification of signs of denervation, fasciculations, myotonia and neuromyotonia in "normal" muscles.

j) Differentiation between cramp and contracture.

Electroneurography consists of the study of nerves, this sensory conduction, motor conduction, $\mathrm{F}$ waves, reflex blink reflex, $\mathrm{H}$ reflex, repetitive stimulation, pudendal nerve protocol, etc... This technique is based on the electrical stimulation of a nerve and the recording of the potential caused by placing electrodes to measure it well in the same nerve, or in the muscle innervated by it. The potential is, therefore, and unlike those spontaneously generated by brain and muscle, an artificially produced potential. Latency, conduction velocity (the time it takes for the potential to occur) to be evaluated; the amplitude, that is to say, the functioning volume of structures that generate the response, and the form thereof, ie the functional integration of said structure.

Latency expresses the degree of myelination of nerve fibers. Increased latency, or even a decrease in nerve conduction velocity, are signs of demyelination. The amplitude of the potential accounts for the number of response generators. At a 
potential recorded on a nerve, the decrease in amplitude is a sign of axon loss. At the potential recorded in a muscle the decrease in amplitude may mean either axons lost, or the inability of the muscle to respond.

Electromiography study of muscle functionality, there are three types of normal, neuropathic and or myopathic patterns.

A. Importance of EMG in different syndromes:

a) Mononeuropathies and entrapment syndromes:

b) Pathophysiology of traumatic nerve injuries (neuroapraxia, axonotmesis neurotmesis

c) Clinical presentation of traumatic injuries:

I. In acute injuries

II. Acute compression injuries

III. Chronic compression injuries

IV. Actrapamientos.

V. EMG diagnosis, classification and prognosis.

VI. Electrophysiological studies in the reinnervation, aberrant axonal regeneration.

VII. Plexopathies and Radiculopathies

VIII. Brachial plexopathy and lumbosacral:

IX. Idiopathic, traumatic, post-radiation

X. Pathophysiology of Radiculopathies

XI. Specific clinical situations

XII. Electrophysiological alterations in radiculopathies. Regional Considerations

XIII. Value and Limitations of Electrophysiological Study

XIV. Intraoperative monitoring

XV. Diseases of motor neurons and spinal cord

Amyotrophic lateral sclerosis, clinical considerations and electrophysiological diagnosis and involvement in the differential diagnosis with motor neuronopathy with anti-GM1 antibodies. Implications of EMG in the diagnosis and assessment of: Poliomyelitis-Post-polio syndrome; viral infections of motor neurons. 2nd focal neuron syndromes: Mononmyelic amyotrophy, Syringomyelia, Congenital and hereditary neuropathies: Clinical pathophysiology and etiopathogenic advances (molecular genetic studies) of neuropathies whose primary polyneurology is the primary or predominant: hereditary sensory-motor neuropathy, neuropathy due to excessive pressure vulnerability, familial amyloidotic polyneuropathies, hereditary sensory and dysautonomic neuropathies, hereditary motor neuronopathies, bulb-spinal neuronopathy linked to the $\mathrm{X}$ chromosome. Electrophysiological studies based on diagnosis. Classification and nosological characterization.

Neuropathies whose polyneuritic syndrome is accompanied by semiologic by affectation of other systems (neurological or non-neurological).

a. Acquired Neuropathies: Physiology - pathophysiology of conduction block, demyelination and axonal degeneration, electroneurographic quantification. Electrophysiological studies in different dysimmune neuropathies (AIDP, CIDP, multifocal motor neuropathy with conduction blockages) performance and limitations in diagnosis, subtype differentiation and prognosis. Characterization of the different forms of diabetic neuropathy. Differential features of EMG results in different toxic and metabolic neuropathies.

b. Neuromuscular transmission disorders: Pathophysiology of neuromuscular transmission. Electrophysiological study in postsynaptic origin processes (myasthenia gravis) its diagnostic performance in the different forms. Study in processes of presynaptic origin (Eaton Lambert syndrome, Botulinum intoxication). Clinical and electrophysiological characterization of the main congenital myasthenic syndromes.

c. Myopathies: Clinical and pathophysiology of genetic processes (Dystrophinopathies sarcoglicanopathies, channelopathies) and acquired (inflammatory, toxic, endocrine myopathies) that affect muscular function. Development and application of quantitative EMG techniques, correlation and support to the indication of histological studies and their limitations.

d. Cranial neuropathy: Assessment and prognosis in facial paralysis, Hemifacial spasm.

B. Syndromes with motor axonal hyperactivity:

a. Central Nervous System:

Electrophysiological study in movement disorders (Tremor, myoclonus, cranio-cervical dystonia and spasm of the clerk ...). Multisystemic atrophy. 
This work is licensed under Creative Commons Attribution 4.0 Licens DOI:_10.19080/OAJS.2017.07.555702

\section{Your next submission with Juniper Publishers} will reach you the below assets

- Quality Editorial service

- Swift Peer Review

- Reprints availability

- E-prints Service

- Manuscript Podcast for convenient understanding

- Global attainment for your research

- Manuscript accessibility in different formats

( Pdf, E-pub, Full Text, Audio)

- Unceasing customer service

Track the below URL for one-step submission https://juniperpublishers.com/online-submission.php 